\title{
Transcriptomic and proteomic analyses of ovarian follicles reveal the role of VLDLR in chicken follicle selection
}

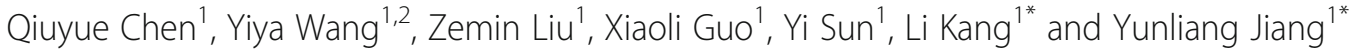

\begin{abstract}
Background: Follicle selection in chickens refers to the process of selecting one follicle from a group of small yellow follicles (SY, 6-8 mm in diameter) for development into 12-15 mm hierarchical follicles (usually F6 follicles), which is an important process affecting laying performance in the poultry industry. Although transcriptomic analysis of chicken ovarian follicles has been reported, integrated analysis of chicken follicles for selection by using both transcriptomic and proteomic approaches is still rarely performed. In this study, we compared the proteomes and transcriptomes of SY and F6 follicles in laying hens and identified several genes involved in chicken follicle selection.

Results: Transcriptomic analysis revealed 855 differentially expressed genes (DEGs) between SY follicles and F6 follicles in laying hens, among which 202 were upregulated and 653 were downregulated. Proteomic analysis revealed 259 differentially expressed proteins (DEPs), including 175 upregulated and 84 downregulated proteins. Among the identified DEGs and DEPs, changes in the expression of seven genes, including VLDLR1, WIF1, NGFR, AMH, BMP15, GDF6 and MMP13, and nine proteins, including VLDLR, VTG1, VTG3, PSCA, APOB, APOV1, F10, ZP2 and ZP3L2, were validated. Further analysis indicated that the mRNA level of chicken VLDLR was higher in F6 follicles than in SY follicles and was also higher in granulosa cells (GCS) than in thecal cells (TCs), and it was stimulated by FSH in GCs.

Conclusions: By comparing the proteomes and transcriptomes of SY and F6 follicles in laying hens, we identified several differentially expressed proteins/genes that might play certain roles in chicken follicle selection. These data may contribute to the identification of functional genes and proteins involved in chicken follicle selection.
\end{abstract}

Keywords: Chicken, Follicle, Proteome, Transcriptome, Differentially expressed genes, Differentially expressed proteins

\section{Background}

The ovary is a dynamic organ and a pivotal component of the reproductive system in hens. In the abdomen of laying hens, ovarian follicles of various sizes exist, including small white follicles that are less than $3 \mathrm{~mm}$ in

\footnotetext{
* Correspondence: kang916@sdau.edu.cn; yljiang723@aliyun.com

'Shandong Provincial Key Laboratory of Animal Biotechnology and Disease Control and Prevention, College of Animal Science and Veterinary Medicine, Shandong Agricultural University, Taian, China

Full list of author information is available at the end of the article
}

diameter, large white follicles that are $3-5 \mathrm{~mm}$ in diameter, small yellow (SY) follicles that are $6-8 \mathrm{~mm}$ in diameter, large yellow follicles that are $9-12 \mathrm{~mm}$ in diameter and five to six hierarchical follicles of increased sizes, i.e., F6 to F1 [1]. Follicle selection in reproductively active domestic hens refers to the daily collection of one follicle from a pool of $6-8 \mathrm{~mm}$ small yellow follicles, which becomes a hierarchical follicle [2] and continues to develop rapidly from the F6 follicle stage to the F1 follicle stage until ovulation. In the process of chicken

(c) The Author(s). 2020 Open Access This article is licensed under a Creative Commons Attribution 4.0 International License, which permits use, sharing, adaptation, distribution and reproduction in any medium or format, as long as you give appropriate credit to the original author(s) and the source, provide a link to the Creative Commons licence, and indicate if changes were made. The images or other third party material in this article are included in the article's Creative Commons licence, unless indicated otherwise in a credit line to the material. If material is not included in the article's Creative Commons licence and your intended use is not permitted by statutory regulation or exceeds the permitted use, you will need to obtain permission directly from the copyright holder. To view a copy of this licence, visit http://creativecommons.org/licenses/by/4.0/ The Creative Commons Public Domain Dedication waiver (http://creativecommons.org/publicdomain/zero/1.0/) applies to the data made available in this article, unless otherwise stated in a credit line to the data. 
follicle selection, granulosa cells rapidly proliferate and differentiate to produce high levels of progesterone $[3,4]$, and vitellin synthesized by the liver enters oocytes via the very low-density lipoprotein receptor (VLDLR) [5, 6].

Changes in the transcripts involved in steroidogenesis, paracrine signaling and transcription during the early stage of follicular growth and development were identified by transcriptome analysis [7]. Comparison among the transcriptomes of small white, F1 and postovulatory chicken follicles identified differentially expressed genes that are involved in the adhesion, apoptosis and steroid biosynthesis pathways [8]. Candidate genes, including ANXA2, Wnt4 and transforming growth factor genes, were shown to play several roles in chicken follicle growth [9-14]. However, the dynamics of the transcriptome during chicken follicle development from the SY follicle to the F6 follicle are unclear.

In addition, the mRNA abundance may not accurately predict the quantities of the corresponding functional proteins, while a proteomic approach can provide a systemic overview of protein levels [15]; therefore, a proteomic approach has certain advantages over mRNA expression profiling [16]. Proteomic analyses of ovarian function [17] and maturation of oocytes [18], such as polycystic ovarian syndrome (PCOS) and cancer [19], in human ovarian diseases and early embryonic development [20-22] in mammals were reported, while in chicken, 2889 proteins were identified in the white yolk and ovarian stroma of small white follicles in Bovan's white laying hen [23]. However, the temporal changes in the proteome during chicken follicle selection are unknown. In this study, we compared the proteomes and transcriptomes of 6-8 $\mathrm{mm}$ SY follicles and the smallest hierarchical follicles (F6) in laying hens and found several differentially expressed genes/proteins (DEGs/DEPs) that might play certain roles in chicken follicle selection.

\section{Results}

\section{Transcriptomic analysis}

RNA-seq was used to compare the transcriptomes of three SY follicles and three of the smallest hierarchical follicles (F6), which are referred to here as S1, S2, and S3 and F1, F2 and F3, respectively. High-throughput
RNA-seq generated $61.66 \mathrm{~Gb}$ of clean data from the six samples of chicken follicles, and $91.07-93.42 \%$ of the reads could be mapped to the chicken genome. For all six samples, at least $93.55 \%$ of the reads were equal to or exceeded Q30 (Table 1).

A total of 855 DEGs, including 202 upregulated and 653 downregulated genes, were identified between the SY follicles (S) and F6 follicles (F) according to the significance criteria of $\mid \log _{2}$ (FoldChange) $\mid>1$ and padj $<$ 0.05 (Fig. 1a). A hierarchical clustered map of DEGs was then constructed and is shown in Fig. 1b. Detailed analysis of the top 10 up-/downregulated DEGs is shown in Table 2. The entire list of DEGs is shown in Table S1.

The DEGs were then assessed by GO and KEGG pathway analyses. The GO functional analysis revealed that most of the DEGs were involved in circulatory system processes, cell differentiation and transition metal ion binding (Fig. 1c). KEGG pathway analysis of the DEGs showed that the most enriched pathways were those involved in TGF- $\beta$ signaling, tyrosine metabolism and cytokine-cytokine receptor interactions (Fig. 1d).

To validate the RNA-seq data, seven DEGs (Table S2), including very low density lipoprotein receptor 1 (VLDLR1), nerve growth factor receptor (NGFR), WNT inhibitory factor 1 (WIF1), anti-Mullerian hormone $(A M H)$, bone morphogenetic protein 15 (BMP15), growth differentiation factor 6 (GDF6) and matrix metallopeptidase 13 (MMP13), were chosen and quantified by quantitative real-time PCR (qRT-PCR). The results showed that the mRNA levels of these genes were similar to those revealed by the sequencing data, suggesting that the RNA-seq results were reliable (Fig. 2).

\section{Proteomics analysis}

The proteins from three SY follicles and three of the smallest hierarchical follicles (F6) that were used for the above transcriptome analysis, i.e., the S1, S2, and S3 and F1, F2 and F3 follicles, were used for TMT labeling and HPLC fractionation followed by LC-MS/MS analysis. The first step was to validate the MS data. The distribution of the mass error was close to zero, and most of the absolute values were less than $5 \mathrm{ppm}$, which meant that the mass accuracy of the MS data was compliant with

Table 1 Summary of the RNA-seq metrics for chicken follicles

\begin{tabular}{lllllc}
\hline Sample & Total reads & Mapped reads, \% & Unique mapped reads, \% & \multicolumn{2}{l}{ GC content, \% } \\
\hline S1 & $62,822,554$ & $57,509,858(91.54 \%)$ & $56,245,873(89.53 \%)$ & 50.83 & 93.75 \\
S2 & $65,638,382$ & $59,775,235(91.07 \%)$ & $58,415,734(89.0 \%)$ & 51.07 & 93.55 \\
S3 & $67,279,424$ & $61,497,217(91.41 \%)$ & $60,160,054(89.42 \%)$ & 50.39 & 93.93 \\
F1 & $66,848,634$ & $61,330,120(91.74 \%)$ & $59,870,822(89.56 \%)$ & 50.65 & 93.71 \\
F2 & $67,659,778$ & $62,284,292(92.06 \%)$ & $60,929,361(90.05 \%)$ & 50.06 & 94.01 \\
F3 & $80,778,754$ & $75,462,807(93.42 \%)$ & $73,776,873(91.33 \%)$ & 94.24 \\
\hline
\end{tabular}




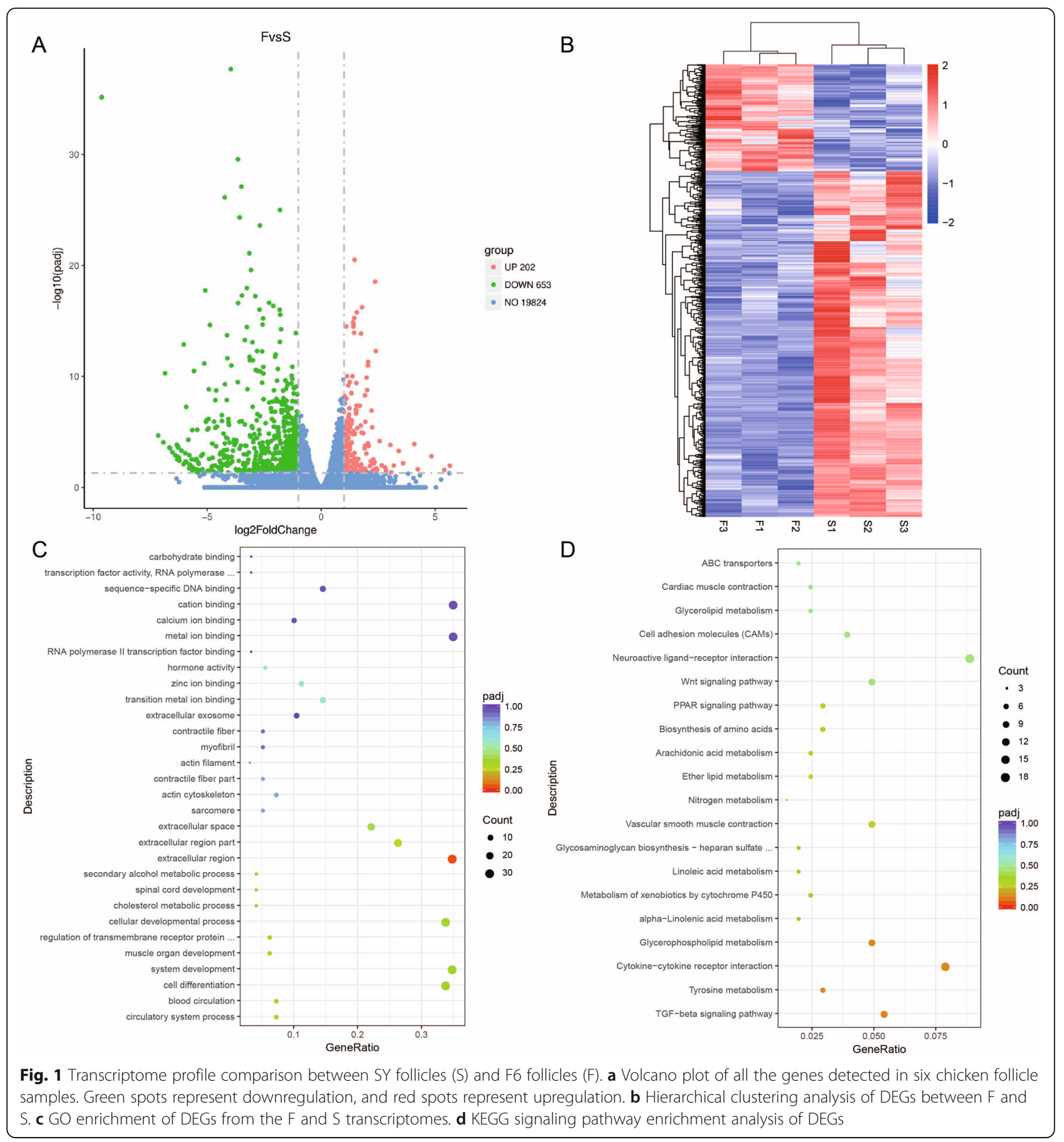

the requirements (Fig. 3a). The length of most peptides was between eight and 16 amino acids, which was in agreement with the general characteristics of tryptic peptides (Fig. 3b). In this study, a total of 5883 proteins were identified in the samples, and 5236 proteins were quantified. According to the relative levels, the quantified proteins were divided into two categories: proteins with a quantitative ratio over 1.5 were considered upregulated, and proteins with a quantitative ratio less than $1 / 1.5$ were considered downregulated $(P<0.05)$ (Fig. 3c). In the F6 follicles, the levels of 175 and 84 proteins were significantly increased and decreased, respectively, compared with those in the SY follicles. A detailed analysis of the top 10 up-/downregulated differentially expressed proteins (DEPs) is shown in Table 3. The entire list of DEPs is shown in Table S3. 
Table 2 The top 10 up- and downregulated genes of chicken F6 vs SY follicles

\begin{tabular}{|c|c|c|c|c|c|}
\hline Gene name & Gene ID & $\log _{2}$ FoldChange & $P$-value & padj & Regulated \\
\hline KRT75L2 & 431,299 & 5.6448359195 & 0.00089581216788 & 0.010968512776 & Up \\
\hline SBK2 & 420,929 & 5.4012005063 & 0.0028916599926 & 0.026749987217 & Up \\
\hline TYRP1 & 395,913 & 4.0884568588 & $3.25 \mathrm{E}-06$ & 0.00011942416133 & Up \\
\hline INHA & 424,197 & 3.5955900397 & 0.0004632196608 & 0.0065938632923 & Up \\
\hline ACTC1 & 423,298 & 3.4047368046 & $1.87 \mathrm{E}-05$ & 0.0005136082463 & Up \\
\hline SPTSSB & 425,008 & 3.1879775126 & 0.00086973054149 & 0.010723329659 & Up \\
\hline DMRT2 & $100,858,556$ & 3.0375474936 & $4.20 \mathrm{E}-06$ & 0.00014842983586 & Up \\
\hline EGR4 & 422,950 & 2.6567614753 & 0.00082143038769 & 0.01027699966 & Up \\
\hline MFSD2B & 428,656 & 2.5659125526 & $1.55 \mathrm{E}-06$ & $6.55 \mathrm{E}-05$ & Up \\
\hline GJD2 & 395,273 & 2.4535441052 & 0.00290562839884 & 0.0268098383411422 & Up \\
\hline SPIRETL & 418,362 & -7.1514399248 & 4.01E-07 & 2.06E-05 & Down \\
\hline SOX3 & 374,019 & -6.9225703884 & $2.13 \mathrm{E}-06$ & $8.52 \mathrm{E}-05$ & Down \\
\hline$M L P H$ & 424,019 & -6.3375597357 & 4.03E-06 & 0.00014300529036 & Down \\
\hline POU4F3 & 395,521 & -6.319423575 & 7.90E-05 & 0.0016173871827 & Down \\
\hline LHX3 & 373,940 & -6.2949552193 & 0.00012211061251 & 0.0022934186943 & Down \\
\hline HAUS3L & $101,751,348$ & -6.1283949694 & $1.72 \mathrm{E}-05$ & 0.00048016708266 & Down \\
\hline GCNT3 & 427,492 & -6.0294054072 & $3.27 \mathrm{E}-16$ & $1.36 \mathrm{E}-13$ & Down \\
\hline GNOT2 & 396,117 & -5.9594390066 & 3.33E-05 & 0.00082793115753 & Down \\
\hline $\mathrm{CDH} 15$ & $107,054,331$ & -5.8252534515 & $1.13 \mathrm{E}-06$ & 5.03E-05 & Down \\
\hline EIF4EIB & $107,054,521$ & -5.7904110653 & $5.48 \mathrm{E}-05$ & 0.0012171943551 & Down \\
\hline
\end{tabular}

The pathways of the DEPs were constructed using KEGG software. Several important pathways were enriched in the F6 follicles compared with the SY follicles (Fig. 3d), including pathways involved in the ribosome, neuroactive ligand-receptor interactions and cytokine-cytokine receptor interactions.

Nine DEPs were randomly selected for parallel reaction monitoring (PRM) analysis to verify the accuracy of the proteome analysis by LC-MS/MS, including apovitellenin1 (APO1), apolipoprotein B (APOB), prostate stem cell antigen (PSCA), coagulation factor X (F10), vitellogenin-1 (VTG1) and vitellogenin-3 (VTG3); all of these proteins were significantly increased in F6 follicles, and zona pellucida sperm-binding protein 2 (ZP2), zona pellucida sperm-binding protein 3 (ZP3) and very low-density lipoprotein receptor (VLDLR) were significantly decreased in F6 follicles (Table 4). The PRM results (Fig. 4) showed that the relative abundances of the peptides from the nine selected individual proteins were consistent with the proteome data.

\section{Transcriptome and proteome association analysis}

The association analysis of the proteomic and transcriptomic data of the F6 and SY follicles revealed a weak relationship between protein and mRNA expression with a

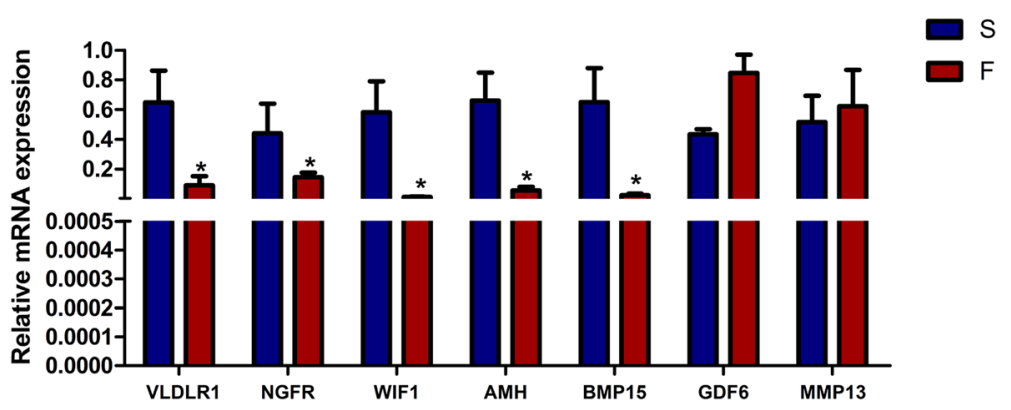

Fig. 2 The mRNA expression levels of genes examined by qRT-PCR. All data are presented as the mean \pm SEM. *,$P<0.05$ 


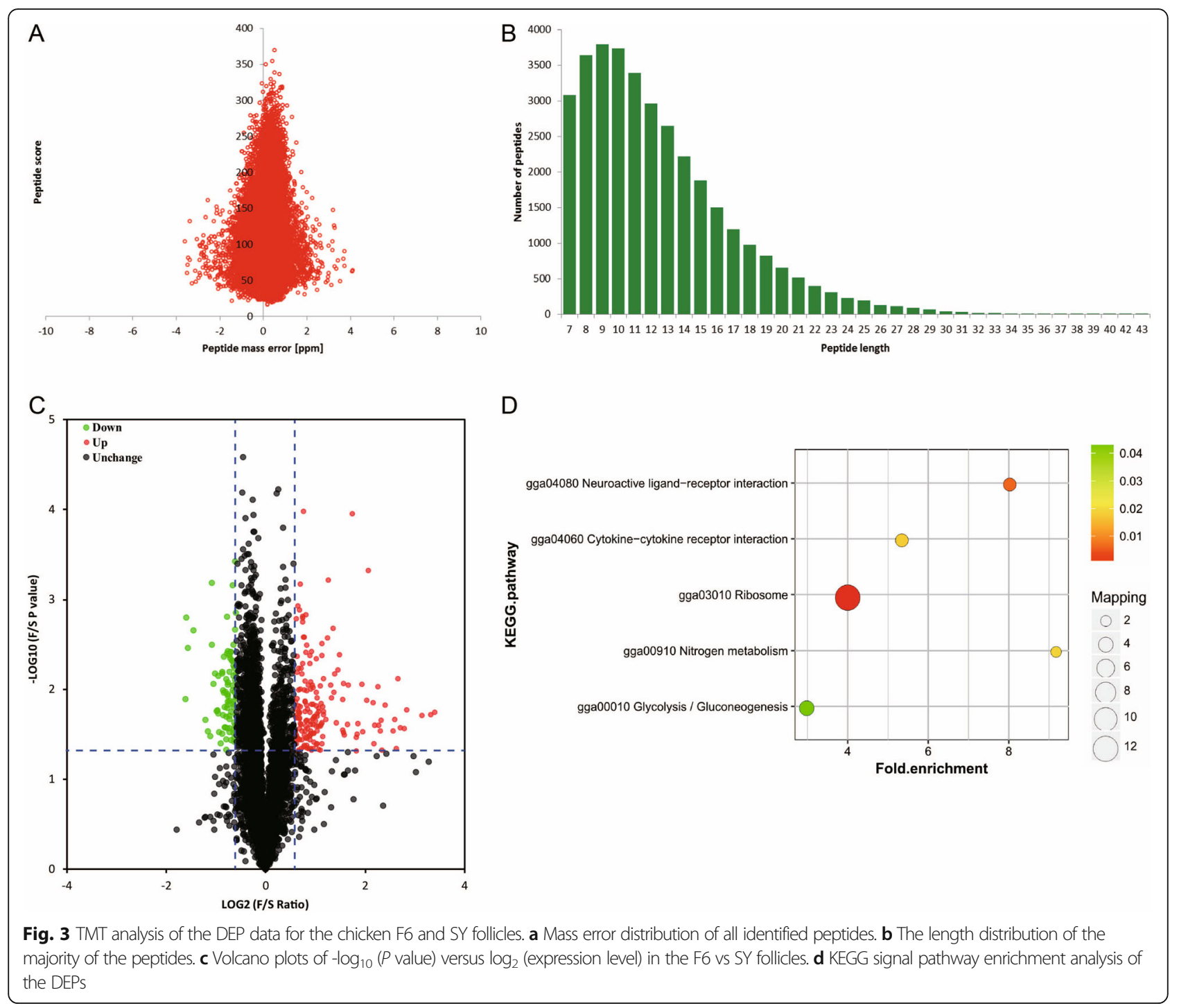

Pearson's correlation coefficient of 0.23 (Fig. 5a). The number of items for which "Transcript up and Protein up" and "Transcript down and Protein down" was 14 $(1.3 \%)$ and 26 (2.4\%), respectively (Fig. 5b). To further understand the relationship between transcripts and proteins, we compared the intersection between DEGs and DEPs (Fig. 6). Most genes were significantly expressed at the mRNA level but not at the protein level. At both the protein and mRNA levels, 14 and 26 genes were revealed as significantly up- and downregulated in SY follicles compared with F6 follicles, respectively. In addition, the expression of two genes were inconsistent in terms of changes in the mRNA levels and protein levels. Table 5 shows the specific regulation information for several genes at the mRNA and protein levels. The specific comparative analysis results are shown in Table S4.

\section{Dynamics and regulation of VLDLR mRNA by FSH}

Both transcriptomic and proteomic analyses indicated that VLDLR expression was significantly downregulated in F6 follicles compared with SY follicles; therefore, we further analyzed the expression of VLDLR mRNA in chicken tissues and found that it was predominantly expressed in the ovary (Fig. 6a), and VLDLR expression in the prehierarchical follicles was significantly higher than that in the hierarchical follicles $(P<0.01)$ (Fig. 6b). In both the hierarchical and prehierarchical follicles, the $V L D L R$ mRNA expression was significantly higher in the granulosa cells $(\mathrm{GCs})$ than in the thecal cells $(\mathrm{TCs})(P<$ $0.05)$ (Fig. 6c). Follicle-stimulating hormone (FSH) treatment stimulated the expression of VLDLR in the GCs, in prehierarchical follicles, the effect was not significant at concentrations of $\leq 10 \mathrm{ng} / \mathrm{ml}$ (Fig. 6d). However, FSH treatment stimulated the expression of $V L D L R$ in the 
Table 3 Top 10 up- and downregulated proteins in chicken F6 vs SY follicles

\begin{tabular}{|c|c|c|c|c|c|}
\hline Protein accession code & Protein description & Protein name & F/S ratio & F/S $P$ value & Regulation \\
\hline P02659 & Apovitellenin-1 & - & 10.52430044 & 0.018057763 & Up \\
\hline A0A1D5NZ61 & Kinesin-like protein KIF20B & - & 9.951417004 & 0.019122892 & Up \\
\hline A0A1D5PYJ4 & Transcriptional repressor p66-alpha & GATAD2A & 8.772228989 & 0.019458417 & Up \\
\hline P41366 & Vitelline membrane outer layer protein 1 & VMO1 & 7.163371488 & 0.016976433 & Up \\
\hline F1NV02 & Apolipoprotein B & APOB & 6.922300706 & 0.026918903 & Up \\
\hline R4GM71 & Phosphatidylcholine-sterol acyltransferase & LCAT & 6.531100478 & 0.026742079 & Up \\
\hline A0A1L1RYU0 & Prostate stem cell antigen & PSCA & 6.297115385 & 0.007551582 & Up \\
\hline A0A1D5NVU2 & Keratin, type II cytoskeletal 75 & KRT75 & 6.183636364 & 0.045556059 & Up \\
\hline P25155 & Coagulation factor $X$ & F10 & 6.062727273 & 0.021845706 & Up \\
\hline A0M8U1 & Suppressor of tumorigenicity 7 protein homolog & ST7 & 5.905829596 & 0.02867129 & Up \\
\hline A0A1L1RJJ7 & Wnt inhibitory factor 1 & WIF1 & 0.327939317 & 0.012827638 & Down \\
\hline A0A1D5P589 & Tudor and $\mathrm{KH}$ domain-containing protein & TDRKH & 0.331994981 & 0.001609664 & Down \\
\hline A0A1D5P0E3 & Epithelial cell adhesion molecule & EPCAM & 0.367965368 & 0.00222955 & Down \\
\hline A0A1D5PS81 & Protein LSM14 homolog B & LSM14B & 0.43363064 & 0.02217556 & Down \\
\hline F1NWH5 & Aquaporin-3 & AQP3 & 0.447158789 & 0.02917623 & Down \\
\hline F1NC54 & SH3 domain and tetratricopeptide repeat-containing protein 1 & SH3TC1 & 0.462576038 & 0.03313208 & Down \\
\hline E1C8L9 & Vacuolar protein sorting-associated protein 29 & VPS29L & 0.475690608 & 0.003217184 & Down \\
\hline C7ACT2 & Unknown & LOC422926 & 0.475784992 & 0.000664487 & Down \\
\hline F1N9X0 & Folate receptor alpha & FOLR1 & 0.482795699 & 0.008705237 & Down \\
\hline F1P337 & Sorting nexin & SNX5 & 0.49547821 & 0.00675724 & Down \\
\hline
\end{tabular}

GCs of hierarchical follicles in a dose-dependent manner $(P<0.01)$ (Fig. 6e).

\section{Discussion}

Follicle selection is an important stage of follicle development that affects many egg production traits in the poultry industry. The mechanism of follicle selection in chickens is becoming a hot topic of research in poultry reproduction biology. Previous studies revealed the effect of Wnt4 [9], bone morphogenetic protein 4 [11], BMP15 [12], $\mathrm{AMH}$ [24], vasoactive intestinal peptide [25] and parathyroid hormone-like hormone [26] on chicken follicle selection and the changes in the RNA N6-methyladenosine methylation profile [27] during chicken follicle selection; however, high-throughput screening of functional genes at the protein level involved in chicken follicle selection is lacking. Therefore, in this study, by combined analysis of changes in the transcriptomic and proteomic profiles of follicles prior to and post selection in chickens, we revealed several DEGs and DEPs, including VLDLR, that may play important roles in chicken follicle selection.

At both the mRNA and protein levels, the expression of VLDLR, NGFR and WIF1 were significantly downregulated in chicken F6 follicles compared with that in SY

Table 4 Nine proteins selected for the parallel reaction monitoring analysis of the chicken follicle proteome data

\begin{tabular}{|c|c|c|c|c|c|c|}
\hline Protein accession code & Protein description & Protein name & Molecular mass (kDa) & F/S ratio & $\mathrm{F} / \mathrm{S} \boldsymbol{P}$ value & $\overline{\text { Regulation }}$ \\
\hline A0A1D5P9N5 & Vitellogenin-1 & VTG1 & 209.88 & 3.858272907 & 0.029564918 & Up \\
\hline A0A1L1RYU0 & Prostate stem cell antigen & PSCA & 13.282 & 6.297115385 & 0.007551582 & Up \\
\hline F1NV02 & Apolipoprotein B & APOB & 523.35 & 6.922300706 & 0.026918903 & Up \\
\hline P02659 & Apovitellenin-1 & APOV1 & 11.966 & 10.52430044 & 0.018057763 & Up \\
\hline P25155 & Coagulation factor $X$ & F10 & 53.141 & 6.062727273 & 0.021845706 & Up \\
\hline Q91025 & Vitellogenin-3 & VTG3 & 38.15 & 4.500719424 & 0.03965785 & Up \\
\hline E1BUH5 & Zona pellucida sperm-binding protein 3 & ZP3 & 51.115 & 0.519704433 & 0.007108315 & Down \\
\hline F1NNU1 & Zona pellucida sperm-binding protein 2 & ZP2 & 77.033 & 0.534041942 & 0.040004135 & Down \\
\hline P98165 & Very low-density lipoprotein receptor & VLDLR & 94.904 & 0.551820728 & 0.019373296 & Down \\
\hline
\end{tabular}




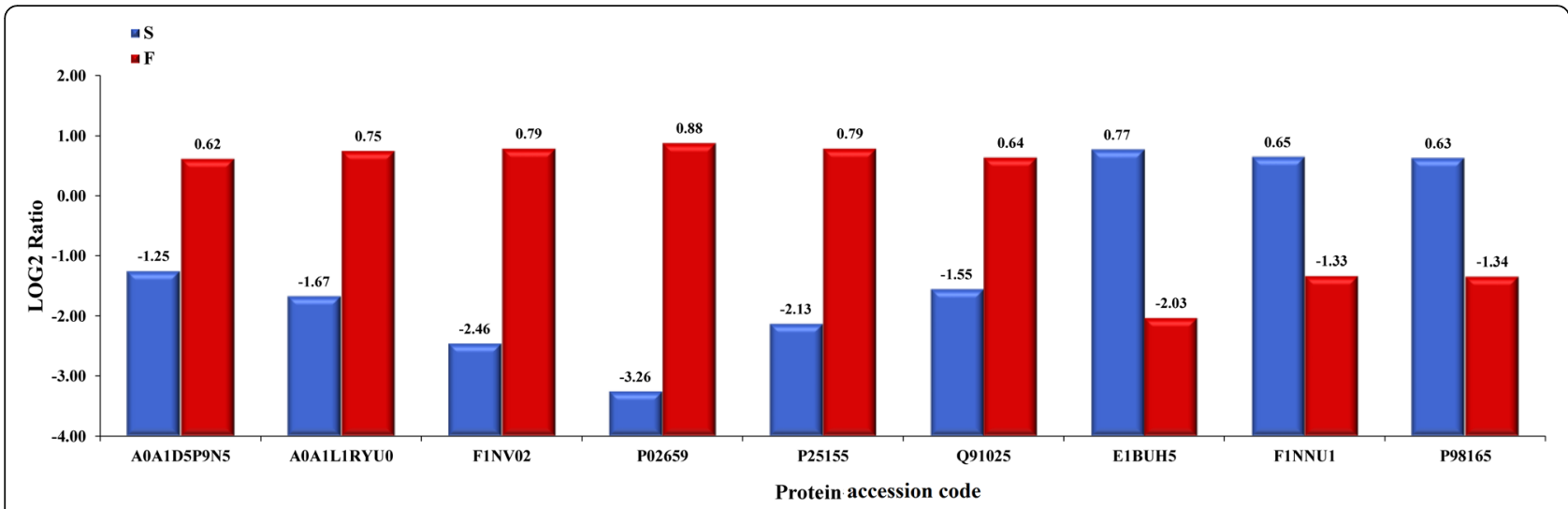

Fig. 4 The histogram of the nine significantly abundant proteins in F6 follicles (F) vs SY follicles $(S)$ according to PRM $(P<0.05)$

follicles. During chicken follicle selection, VLDLR plays a pivotal role in the absorption of vitelin by oocytes, and without VLDLR, oocytes are unable to enter the rapid growth stage of follicle development [28]. During the development of the small white follicle, VLDLR migrates to the follicular wall, enabling the endocytosis of vitellogenin into the yolk, followed by follicular differentiation [7]. Studies have revealed that the expressed variant of $V L D L R$ in chicken granulosa cells differs from the variant expressed in the oocyte, which contains an O-linked sugar domain (VLDLR 1) [29, 30], and the reduced level of VLDLR in granulosa cells is suggested to allow more VLDLR to reach the oocytes by passing through intercellular gaps rather than via receptor-mediated endocytosis into granulosa cells [31]. In this study, we provided further evidence that after follicle selection, the expression of $V L D L R$ was significantly decreased. Moreover, we found that $V L D L R$ is mainly expressed in chicken ovaries, SW and SY follicles and the GCs of prehierarchical follicles, and its expression was stimulated by FSH in the GCs, especially in those of hierarchical follicles. These data collectively suggest that, after follicle selection, the decreased expression of VLDLR in GCs might allow more VLDLR 1 to be expressed on the oocyte membrane, thus promoting the rapid growth of hierarchical follicles. Similarly, in geese, the expression of VLDLR mRNA is decreased concomitant with an increase in the follicular diameter [32]. For NGFR, in human mural and cumulus granulosa cells, the nerve growth factor receptor tropomyosin-related kinase A (TrkA) mRNA level was strongly correlated with the number of oocytes retrieved, and the number of oocytes retrieved was greater among women with a low p75(NTR)/TrkA ratio [33]. The role of WIF1 has not been reported in ovarian
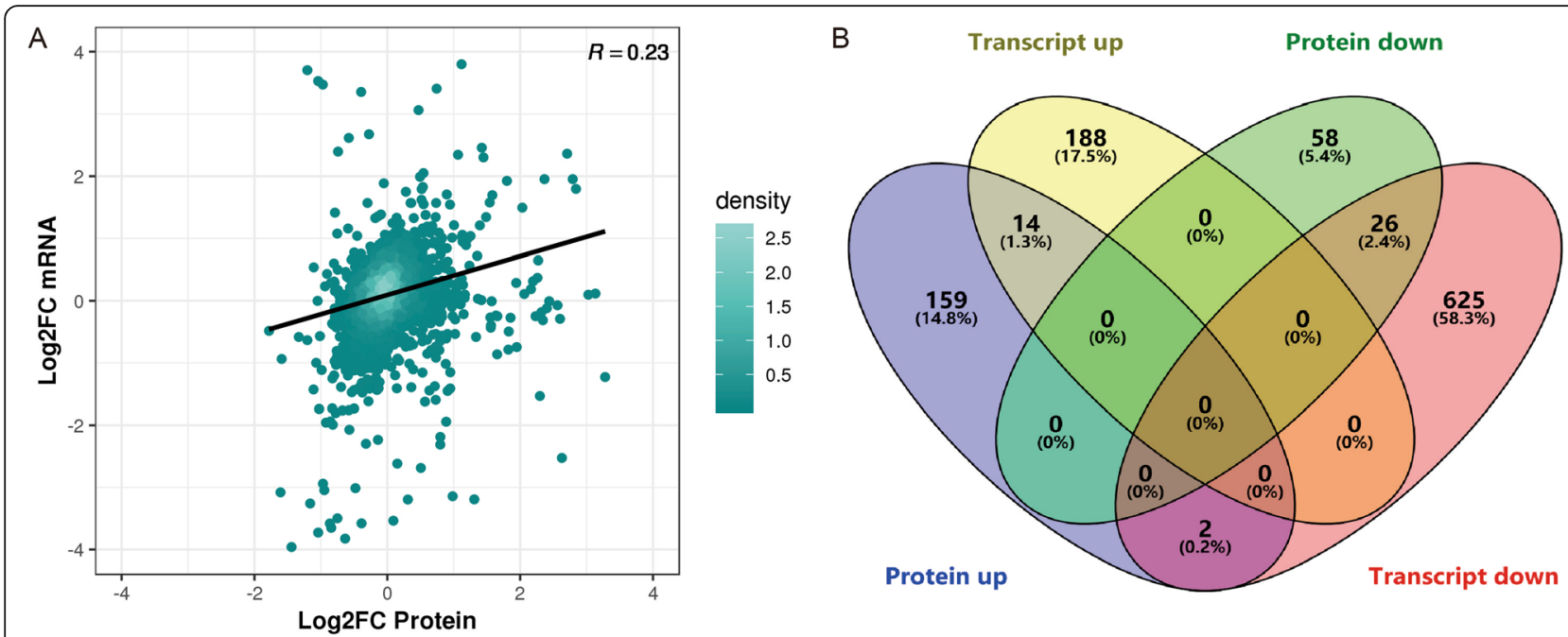

Fig. 5 Association analysis (a) and Venn diagram (b) of differentially expressed genes/proteins from the TMT and DEG analyses between F6 and SY follicles in laying hens 


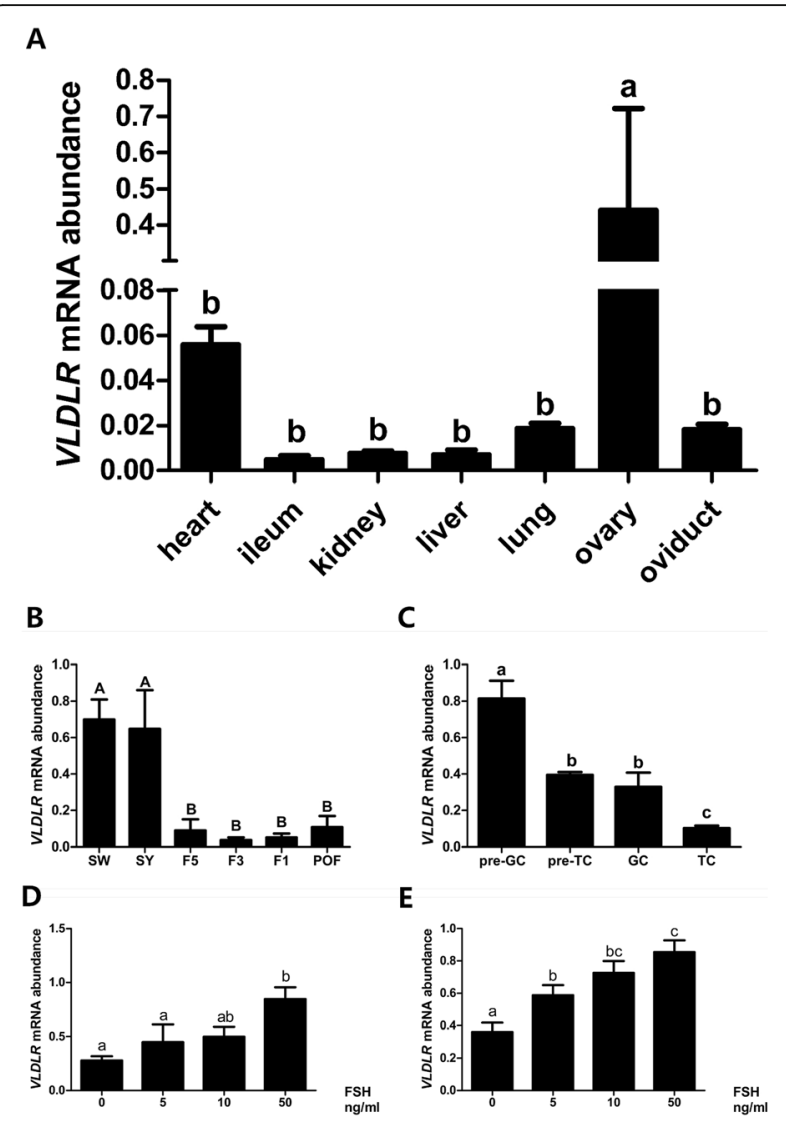

Fig. 6 Dynamics of the expression of chicken VLDLR mRNA and the effect of follicle-stimulating hormone (FSH) treatment on the VLDLR mRNA levels in the granulosa cells (GCs) of chicken ovarian follicles. a Expression of VLDLR mRNA in different chicken tissues. $\mathbf{b}$ Expression levels of VLDLR in 1-2 $\mathrm{mm}$ follicles, 6-8 $\mathrm{mm}$ follicles (small yellow follicles), the fifth largest follicles (F5), the third largest follicles (F3), the largest follicles (F1), and the new postovulatory follicles (POFs). $\mathbf{c}$ Expression of chicken $V L D L R$ in the granulosa cells (pre-GCs) and theca cells (pre-TCs) of prehierarchical follicles and the GCs and TCs of hierarchical follicles. $\mathbf{d}$ Effect of FSH on VLDLR in the GCS of prehierarchical follicles. e Effect of FSH on VLDLR in the GCs of hierarchical follicles. All data are presented as the mean \pm SEM. $\left({ }^{a b c} p<0.05 ;{ }^{A B C} p<0.01\right)$

function or follicle growth. The function of NGFR and WIF1 in chicken follicle selection requires further investigation.

Hundreds of DEGs were identified by transcriptome comparison between SY and F6 follicles during chicken follicle selection. Among these DEGs, the expression of $\mathrm{AMH}$ and BMP15 was greatly decreased in F6 follicles compared with that in SY follicles. Similar dynamics for $\mathrm{AMH}$ and BMP15 expression were previously reported; $\mathrm{AMH}$ is mainly expressed in granulosa cells of $1-5 \mathrm{~mm}$ follicles in the early stage of follicular development [1] and is decreased in follicles after selection [24], while BMP15 may promote follicle selection and affect granulosa cell proliferation and steroidogenesis in hens [12].
Among the 259 DEPs identified by global proteome analysis of chicken SY and F6 follicles, the following proteins may play certain roles in chicken follicle selection. APO1 is one of five apoproteins that forms low-density lipoprotein (LDL) and is expressed in the egg yolk and vitelline membrane $[34,35]$. APOB is the major apolipoprotein in very low-density lipoprotein (VLDL) and LDL and is a ligand for LDL receptors. The increased expression of APOV1 and APOB in F6 follicles is consistent with higher yolk incorporation after follicle selection. Vitellogenins are yolk precursor proteins produced by the liver and are essential for the growth of chicken ovarian follicles, and they circulate in the bloodstream until a follicle enters the stage of vitellogenesis, which triggers endocytosis of vitellogenins and transport into the yolk [6]. Vitellogenins 1, 2, and 3 were found in chicken egg yolk plasma and granules [35]. The increased expression of VTG1 and VTG3 in F6 follicles found in this study suggests that yolk deposition is active in F6 follicles. The zona pellucida (ZP) is a specialized extracellular matrix that surrounds the oocyte and early embryo and is composed of three or four glycoproteins, including zona pellucida sperm-binding proteins 1-4 (ZP1-4), with various functions in oogenesis and fertilization [36]. ZP3 has been shown to promote oocyte maturation in pigs [37]. In this study, we found that the expression levels of ZP2 and ZP3 were decreased to approximately half of the level found in F6 follicles; the mechanism of this process in follicle selection requires further study.

In this study, by association analysis between the transcriptome and the proteome, we found that the expression changes of most genes are inconsistent at the mRNA and protein levels, which is in line with the results of other studies showing that most genes can exhibit differential expression at the transcript and protein levels $[38,39]$, and this suggests that it is necessary to analyze the functions of genes at both levels.

\section{Conclusions}

By comparing the transcriptomes and proteomes of SY follicles and F6 follicles in laying hens, this study revealed 855 DEGs and 259 DEPs. The possible functional significance of several DEGs and DEPs, including VLDLR, was further discussed. These data may contribute to the identification of the functional genes and proteins involved in chicken follicular development and selection.

\section{Methods}

\section{Tissue collection}

Fifteen randomly sampled Hy-line brown hens from the same batch, which had been laying regularly for at least 1 month (28 weeks old, with a mean body weight of 
Table 5 Differentially expressed genes at both the mRNA and protein levels between SY and F6 follicles in chicken

\begin{tabular}{|c|c|c|c|c|c|c|}
\hline \multirow[t]{2}{*}{ Gene } & \multicolumn{3}{|l|}{ Transcript } & \multicolumn{3}{|l|}{ Protein } \\
\hline & $\log _{2}$ FoldChange & FDR & Regulation type & F/S ratio & $P$-value & Regulation type \\
\hline$\overline{V L D L R}$ & -1.93821454837682 & 0.000000000251 & Down & 0.552 & 0.0194 & Down \\
\hline NGFR & -1.99046510645846 & $1.07 \mathrm{E}-12$ & Down & 0.566 & 0.0264 & Down \\
\hline WIF1 & -3.079759991 & $2.56 \mathrm{E}-20$ & Down & 0.328 & 0.0128 & Down \\
\hline KPNA7 & -5.083462853 & $1.76 \mathrm{E}-18$ & Down & 0.581 & 0.0362 & Down \\
\hline ACTC1 & 3.404736805 & 0.000513608 & UP & 1.673 & 0.00158 & UP \\
\hline LCAT & 2.363270714 & 2.85E-19 & UP & 6.531 & 0.0267 & UP \\
\hline$Z P D$ & 2.302937808 & 0.007012137 & UP & 2.727 & 0.0061 & UP \\
\hline ACTA1 & 1.543125393 & 0.000000000407 & UP & 1.923 & 0.00953 & UP \\
\hline$A G T$ & -3.192564345 & 0.003115348 & Down & 2.478 & 0.00904 & UP \\
\hline A2 ML1 & -1.15166374 & 0.000215557 & Down & 1.929 & 0.0304 & UP \\
\hline
\end{tabular}

$2.1 \pm 0.12 \mathrm{~kg}$ ) and were housed under standard conditions with free access to food and water, were divided into three biological groups, each containing five hens. Vaccination was performed according to the recommendations from Hy-line International. The experimental hens were killed by cervical dislocation after approximately $10 \mathrm{~h}$ after laying, the time of which was individually recorded. From each hen, small yellow follicles $(6-8$ $\mathrm{mm}$ in diameter, SY) and smallest hierarchical follicle (12-15 mm in diameter, F6) were separately collected, and the egg yolk was carefully squeezed out with tweezers, washed with phosphate-buffered saline (Thermo Fischer Scientific, MA, USA), immediately frozen in liquid nitrogen and used for transcriptomic and proteomic analyses. The Institutional Animal Care and Use Ethics Committee of Shandong Agricultural University reviewed and approved all procedures described in this study. This study was performed according to the Guidelines for Experimental Animals of the Ministry of Science and Technology of China. Three biological replicates were prepared for the transcriptomic and proteomic analyses of total RNA and proteins.

\section{Transcriptome analysis}

Total RNA was isolated from follicles and follicular granulosa and thecal cells with TRIzol reagent (Invitrogen, CA, USA). The purity and integrity of total RNA were checked using the NanoPhotometer ${ }^{\circ}$ spectrophotometer (IMPLEN, CA, USA) and the RNA Nano 6000 Assay Kit of the Bioanalyzer 2100 system (Agilent Technologies, CA, USA), respectively. Sequencing libraries were generated using NEBNext ${ }^{\circ}$ UltraTM RNA Library Prep Kit for Illumina ${ }^{\circ}$ (NEB, USA) following manufacturer's recommendations and index codes were added to attribute sequences to each sample. The library preparations were sequenced on an Illumina Novaseq platform. Clean data (clean reads) were obtained by removing reads containing adapter, reads containing ploy- $\mathrm{N}$ and low quality reads from raw data. The index of the reference genome was built and the paired-end clean reads were aligned to the Gallus gallus genome (ftp://ftp.ncbi. nlm.nih.gov/genomes/all/GCF/000/002/315/GCF 000002315.6_GRCg6a/) using Hisat2 v2.0.5 [40]. Gene expression level was quantified with featureCounts v1.5.0-p3 and FPKM [41].

Differentially expressed genes (DEGs) between SY and F6 follicles were identified according to the cretiera of adjusted $P$-value $<0.05$ and a $\mid \log _{2}$ FoldChange $\mid>1$ according to DESeq2 [42]. Gene ontology (GO) enrichment analysis and Kyoto Encyclopedia of Genes and Genomes database (KEGG) pathway enrichment analysis of the DEGs were implemented by the cluster Profiler R package, and genes with $P$-values less than 0.05 were considered significantly enriched as differentially expressed [43]. The transcriptome data have been deposited with the NCBI Sequence Read Archive (SRA, https://www.ncbi.nlm.nih. gov/sra/docs/) under accession number SRP236909.

\section{Sample processing and liquid chromatography coupled with tandem mass spectrometry (LC-MS/MS)}

The proteins in the tissues were extracted with lysis buffer containing $8 \mathrm{M}$ urea (Sigma Aldrich, MO, USA) and $1 \%$ protease inhibitor cocktail (Merck Millipore, MA, USA), their concentration was determined with a BCA kit (Beyotime, Shanghai, China) according to the manufacturer's instructions. Protein enzymolysis was performed using trypsin (Promega, WI, USA). The peptides were desalted on a Strata X C18 SPE column (Phenomenex, CA, USA) and vacuum-dried after trypsin digestion, reconstituted in $0.5 \mathrm{M}$ TEAB and processed according to the manufacturer's protocol for the TMT kit (Thermo Fischer Scientific, MA, USA). The tryptic peptides were fractionated by high-pH reverse-phase HPLC using an Agilent 300 Extend C 18 column $(5 \mu \mathrm{m}$ particle size, $4.6 \mathrm{~mm}$ ID, $250 \mathrm{~mm}$ length), dissolved in $0.1 \%$ formic acid (Sigma Aldrich, MO, USA) (solvent A) 
and separated using EASY-nLC 1000 ultra-high performance liquid phase system. Finally, peptides that were separated were exposed to an NSI source followed by tandem mass spectrometry (MS/MS) with a Q Exactive ${ }^{\mathrm{Tm}}$ Plus spectrometer (Thermo Fischer Scientific, MA, USA) coupled online to the UPLC system.

The resulting MS/MS data were processed using the Maxquant search engine (v.1.5.2.8). The tandem mass spectra were searched against the Gallus gallus database (http://www.uniprot.org/proteomes/UP000000539, chicken proteome ID: UP000000539) concatenated with the reverse decoy database. The GO proteome was derived from the UniProt-GOA database (http://www.ebi. ac.uk/GOA/). The KEGG database was used to identify the enriched pathways. Proteins with a threshold of $P<$ 0.05 and a fold change of $>1.5$ or $<1 / 1.5$ were identified as differentially expressed proteins (DEPs) between SY and F6 follicles. The MS proteomics data have been deposited in the ProteomeXchange Consortium (http:// www.proteomex change.org/) via the PRIDE partner repository with the dataset identifier PXD011470 (Username: reviewer39674@ebi.ac.uk, Password: AfwLJZRb).

\section{Parallel reaction monitoring}

PRM used to validate protein abundance levels of APO1, APOB, PSCA, F10, VTG1 and VTG3 that were significantly increased in F6 follicles, and ZP2, ZP3 and VLDLR that were significantly decreased in F6 follicles obtained from TMT analysis. PRM analysis was carried out at the Jingjie PTM BioLab Co., Ltd. (Hangzhou, China) with experimental steps and parameter settings used according to the reference [44].

\section{Cell culture and cell treatment}

Granulosa and thecal cells were prepared according to references $[14,45]$, respectively. Firstly, the yolk was carefully removed with ophthalmic forceps. Then, small yellow follicles were digested with $0.1 \%$ collagenase II (MP Biomedicals, Santa Ana, CA, USA) at $38^{\circ} \mathrm{C}$ for $15 \mathrm{~min}$ to obtain the pre-GCs, for additional $30 \mathrm{~min}$ to obtain the pre-TCs. For hierarchical follicles, after the removal of yolk, granulosa cells were firstly separated from the theca externa cells with ophthalmic forceps, followed by treatent with $0.25 \%$ trypsin-EDTA (Gibco-BRL, NY, USA) at $38^{\circ} \mathrm{C}$ for 15 min, while the thecal cells were obtained by digesting the theca externa and interna with $0.1 \%$ collagenase II at $38{ }^{\circ} \mathrm{C}$ for $30 \mathrm{~min}$. After centrifugation, the cells were suspended in culture medium containing M199 (Gibco-BRL, NY, USA), 10\% fetal bovine serum (Biological Industries, Kibbutz Beit Haemek, Israel) and $1 \%$ penicillin/streptomycin (Solarbio, Beijing, China) and subsequently seeded at a density of $1 \times 10^{5}$ cells/ well in 24-well culture plates and cultured at $38^{\circ} \mathrm{C}$ in a water-saturated atmosphere of $95 \%$ air and $5 \% \mathrm{CO}_{2}$. The number of viable cells was estimated using Trypan blue staining. After $24 \mathrm{~h}$, cultured granulosa cells were treated with different concentrations $(0,5,10$, and $50 \mathrm{ng} / \mathrm{ml}$ ) of recombinant FSH (R\&D Systems, MN, USA). All the treated cells were collected after another $24 \mathrm{~h}$ for RNA extraction and qRT-PCR analysis.

\section{Real-time quantitative PCR}

Total RNA was extracted from chicken ovarian follicles that were also used for proteome analysis using TRIzol reagent (InvivoGen, CA, USA). Synthesis of the cDNA was performed using a PrimeScript RT reagent kit with $1 \mu \mathrm{g}$ of the RNA pretreated with gDNA Eraser (TaKaRa, Dalian, China) according to the manufacturer's protocol. Real-time quantitative PCR of the mRNA expression level of VLDLR, VLDLR1, WIF1, NGFR, AMH, BMP15, GDF6 and MMP13 was performed using a SYBR Premix Ex $\mathrm{Taq}^{\text {TM }}$ II kit (TaKaRa, Dalian, China) with primers listed in Table S5 on a Light Cycler 480 real-time PCR system (Roche, Basel, Switzerland) as follows: $95^{\circ} \mathrm{C}$ for $30 \mathrm{~s}$, followed by 40 cycles of denaturation at $95^{\circ} \mathrm{C}$ for $10 \mathrm{~s}$ and annealing and extension at $58^{\circ} \mathrm{C}$ for $20 \mathrm{~s}$. The melting curves were obtained, and quantitative analysis of the data was performed using the $2^{-\Delta \Delta C T}$ relative quantification method [46]. Quantification was performed by standardizing the reaction results versus those of $\beta$-actin.

\section{Statistical analysis}

Differences in mRNA expression between follicles and follicular cells were evaluated by one-way ANOVA followed by Duncan's multiple range test $(P<0.05)$ using the General Linear Model procedure in SAS (version 9.2). For one experiment, each treatment was repeated four times, and at least three independent experiments were performed. All data are presented as the mean \pm SEM $(n=4)$. The GO and KEGG analyses were performed by using Fisher's t-test. $P<0.05$ was considered to indicate a significant difference.

\section{Supplementary information}

Supplementary information accompanies this paper at https://doi.org/10. 1186/s12864-020-06855-w.

Additional file 1: Table S1. DEGs. (XLS $315 \mathrm{~kb}$ )

Additional file 2: Table S2. Seven DEGs selected for GRT-PCR validation in chicken follicles. (DOCX 13kb)

Additional file 3: Table S3. DEPs between F and S. (XLSX 171 kb)

Additional file 4: Table S4. Protein and Transcript quantiation combine. (XLS $35 \mathrm{~kb}$ )

Additional file 5: Table S5. The primers used in the

experiments. (DOCX 20kb) 


\section{Abbreviations}

BP: Biological process; CC: Cellular component; DEGs: Differentially expressed genes; DEPs: Differentially expressed proteins; GO: Gene ontology; KEGG: Kyoto Encyclopedia of Genes and Genomes; GCs: granulosa cells; TCs: thecal cells

\section{Acknowledgments}

The authors thank PTM-Biolabs Co., Ltd. (Hangzhou, China) for mass spectrometry analysis and help in preparing the manuscript. The authors would also like to thank Novogene Bioinformatics Technology Co., Ltd. (Beijing, china) for their bioinformatics supports.

\section{Authors' contributions}

QC, LK and YJ designed and drafted the manuscript. QC, YW and ZL carried out animal care, prepared samples and performed the experiments. QC and XG performed the data processing and biological information analysis. YJ, LK and YS conceived the study and the experimental design and helped draft the manuscript. All authors read and approved the final manuscript.

\section{Funding}

This research was financially supported by grants from the National Natural Science Foundation of China (NSFC 31672414, 31272435, 31772588), the Funds of Shandong"Double Tops" Program (SYL2017YSTD12) and Shandong Agricultural Breed Project (2019LZGC019). The funding body had no role in the design of the study and collection, analysis, and interpretation of data and in writing the manuscript.

\section{Availability of data and materials}

The reference genome (ftp://ftp.ncbi.nlm.nih.gov/genomes/all/GCF/000/ 002/315/GCF_000002315.6_GRCg6a/) and proteome (http://www.uniprot. org/proteomes/ UP000000539, chicken proteome ID: UP000000539) database of the Gallus gallus were used in this study. The transcriptome data have been deposited with the NCBI Sequence Read Archive (SRA, https://www. ncbi.nlm.nih.gov/sra/docs/) under accession number SRP236909. The MS proteomics data have been deposited in the ProteomeXchange Consortium (http://www.proteomex change.org/) via the PRIDE partner repository with the dataset identifier PXD011470 (Username: reviewer39674@ebi.ac.uk, Password: AfwLJZRb).

\section{Ethics approval and consent to participate}

The animal experiments were carried out in accordance with the protocols of the 'Guidelines for Experimental Animals' of the Ministry of Science and Technology (Beijing, China) and all efforts were made to minimize suffering. The animal experiments were approved by the Institutional Animal Care and Use Ethics Committee of Shandong Agricultural University (Permit Number: $2,007,005)$. We have obtained the informed consents of a written form from Mr. Xiaoyan Yang who was the owner of the Hy-line brown hens.

\section{Consent for publication}

Not applicable.

\section{Competing interests}

The authors declare that they have no competing interests.

\section{Author details}

${ }^{1}$ Shandong Provincial Key Laboratory of Animal Biotechnology and Disease Control and Prevention, College of Animal Science and Veterinary Medicine, Shandong Agricultural University, Taian, China. ${ }^{2}$ College of Life Science, Qi Lu Normal University, Jinan, China.

Received: 2 November 2019 Accepted: 19 June 2020

Published online: 16 July 2020

\section{References}

1. Johnson AL. Ovarian follicle selection and granulosa cell differentiation. Poult Sci. 2014:94(4):781-5.

2. Onagbesan O, Bruggeman V, Decuypere E. Intra-ovarian growth factors regulating ovarian function in avian species: a review. Anim Reprod Sci. 2009;111:121-40.
3. Tilly JL, Kowalski Kl, Johnson AL. Stage of ovarian follicular development associated with the initiation of steroidogenic competence in avian granulosa cells. Biol Reprod. 1991;44(2):305-14.

4. Johnson AL, Woods DC. Ovarian dynamics and follicle development. In: Jamieson BGM, editor. Reproductive biology and phylogeny of birds. Enfield (NH): Science Publishers, an imprint of Edenbridge Ltd. 2007; pp: 243-277 (Chapter 6).

5. Stifani S, Barber DL, Nimpf J, Schneider W. A single chicken oocyte plasma membrane protein mediates uptake of very low density lipoprotein and vitellogenin. Proc Natl Acad Sci. 1990;87:1955-9.

6. Schneider W. Receptor-mediated mechanisms in ovarian follicle and oocyte development. Gen Comp Endocrinol. 2009;163:18-23.

7. Diaz FJ, Anthony K, Halfhill AN. Early avian follicular development is characterized by changes in transcripts involved in steroidogenesis, paracrine signaling and transcription. Mol Reprod Dev. 2011;78:212-23.

8. Zhu G, Mao Y, Zhou W, Jiang Y. Dynamic changes in the follicular transcriptome and promoter DNA methylation pattern of steroidogenic genes in chicken follicles throughout the ovulation cycle. PLoS One. 2015; 10:e0146028.

9. Wang Y, Chen Q, Liu Z, Guo X, Du Y, Yuan Z, Guo M, Sun Y, Kang L, Jiang Y. Transcriptome analysis on single small yellow follicles reveals that WNT4 is involved in chicken follicle selection. Front Endocrinol. 2017;8:317.

10. Ocón-Grove OM, Poole DH, Johnson AL. Bone morphogenetic protein 6 promotes FSH receptor and anti-müllerian hormone mRNA expression in granulosa cells from hen prehierarchal follicles. Reproduction. 2012;14:3825.

11. Kim D, Ocón-Grove O, Johnson AL. Bone morphogenetic protein 4 supports the initial differentiation of hen (Gallus gallus) granulosa cells. Biol Reprod. 2013;88:161.

12. Stephens $\mathrm{CS}$, Johnson PA. Bone morphogenetic protein 15 may promote follicle selection in the hen. Gen Comp Endocrinol. 2016;235:170-6.

13. Knight PG, Glister C. TGF-beta superfamily members and ovarian follicle development. Reproduction. 2016;132:191-206.

14. Zhu G, Chen X, Mao Y, Kang L, Ma X, Jiang Y. Characterization of annexin A2 in chicken follicle development: evidence for its involvement in angiogenesis. Anim Reprod Sci. 2015;161:104-11.

15. Ewen K, Baker M, Wilhelm D, Aitken RJ, Koopman P. Global survey of protein expression during gonadal sex determination in mice. Mol Cell Proteomics. 2009:8:2624

16. Ma M, Guo X, Wang F, Zhao C, Liu Z, Shi Z, Wang Y, Zhang P, Zhang K, Wang $N$, et al. Protein expression profile of the mouse metaphase-ii oocyte J Proteome Res. 2008;7:4821-30.

17. Wang L, Zhu YF, Guo XJ, Huo R, Ma X, Lin M, Zhou ZM, Sha JH. A twodimensional electrophoresis reference map of human ovary. J Mol Med. 2005:83:812-21.

18. Pfeiffer MJ, Siatkowski M, Paudel Y, Balbach ST, Baeumer N, Crosetto N, Drexler HC, Fuellen G, Boiani M. Proteomic analysis of mouse oocytes reveals 28 candidate factors of the "reprogrammome". J Proteome Res. 2011;10:2140-53.

19. Upadhyay RD, Balasinor NH, Kumar AV, Sachdeva G, Parte P, Dumasia K. Proteomics in reproductive biology: beacon for unraveling the molecular complexities. Biochim Biophys Acta. 1834;2013:8-15.

20. Massicotte L, Coenen K, Mourot M, Sirard MA. Maternal housekeeping proteins translated during bovine oocyte maturation and early embryo development. Proteomics. 2006;6:3811-20.

21. Memili E, Peddinti D, Shack LA, Nanduri B, McCarthy F, Sagirkaya H, Burgess SC. Bovine germinal vesicle oocyte and cumulus cell proteomics. Reproduction. 2007;133:1107-20.

22. Peddinti D, Memili E, Burgess SC. Proteomics-based systems biology modeling of bovine germinal vesicle stage oocyte and cumulus cell interaction. PLoS One. 2010:5:e11240

23. Nepomuceno Al, Muddiman DC, Petitte JN. Global proteomic analysis of functional compartments in immature avian follicles using laser microdissection coupled to LC-MS/MS. J Proteome Res. 2015;14:3912-23.

24. Johnson PA. Follicle selection in the avian ovary. Reprod Domest Anim. 2012;47(Suppl 4):283-7.

25. Kim D, Johnson AL. Vasoactive intestinal peptide promotes differentiation and clock gene expression in granulosa cells from prehierarchal follicles. Mol Reprod Dev. 2016;83(5):455-63.

26. Guo X, Wang Y, Chen Q, Yuan Z, Chen Y, Guo M, Kang L, Sun Y, Jiang Y. The role of $\mathrm{PTHLH}$ in ovarian follicle selection, its transcriptional regulation and genetic effects on egg laying traits in hens. Front Genet. 2019;10:430. 
27. Fan Y, Zhang C, Zhu G. Profiling of RNA N6-methyladenosine methylation during follicle selection in chicken ovary. Poult Sci. 2019;98(11):6117-24.

28. Nimpf J, Radosavljevic MJ, Schneider WJ. Oocytes from the mutant restricted ovulator hen lack receptor for very low density lipoprotein. J Biol Chem. 1989;264:1393-8.

29. Hayashi K, Nimpf J, Schneider WJ. Chicken oocytes and fibroblasts express different apolipoprotein-B-specific receptors. J Biol Chem. 1989;264:3131-9.

30. Eresheim C, Leeb C, Buchegger P, Nimpf J. Signaling by the extracellular matrix protein reelin promotes granulosa cell proliferation in the chicken follicle. J Biol Chem. 2014;289:10182-91.

31. Lin X, Ma Y, Qian T, Yao J, Mi Y, Zhang C. Basic fibroblast growth factor promotes prehierarchical follicle growth and yolk deposition in the chicken. Theriogenology. 2019;139:90-7.

32. Hu S, Liu H, Pan Z, Xia L, Dong X, Li L, Xu F, He H, Wang J. Molecular cloning, expression profile and transcriptional modulation of two splice variants of very low density lipoprotein receptor during ovarian follicle development in geese (Anser cygnoide). Anim Reprod Sci. 2014;149:281-96.

33. Buyuk E, Santoro N, Cohen HW, Charron MJ, Jindal S. Reduced neurotrophin receptor tropomyosin-related kinase a expression in human granulosa cells: a novel marker of diminishing ovarian reserve. Fertil Steril. 2011;96(2):474-8.

34. Mann K. Proteomic analysis of the chicken egg vitelline membrane. Proteomics. 2008:8:2322-32.

35. Mann K, Mann M. The chicken egg yolk plasma and granule proteomes. Proteomics. 2010;8:178-91.

36. Wassarman PM, Jovine L, Qi H, Williams Z, Darie C, Litscher ES. Recent aspects of mammalian fertilization research. Mol Cell Endocrinol. 2005;234: 95-103.

37. Sakaguchi Y, Uzuhashi R, Iwata H, Monji Y, Kuwayama T. Changes in the sperm-zona pellucida binding properties during porcine oocyte maturation. J Mammalian Ova Res. 2010;27:130-5.

38. Song J, Wang C. Transcriptomic and proteomic analyses of genetic factors influencing adductor muscle coloration in QN Orange scallops. BMC Genomics. 2019;20:363.

39. Abdulghani M, Song G, Kaur H, Walley JW, Tuteja G. Comparative analysis of the transcriptome and proteome during mouse placental development. J Proteome Res. 2019;18(5):2088-99.

40. Kim D, Langmead B, Salzberg SL. HISAT: a fast spliced aligner with low memory requirements. Nat Methods. 2015;12(4):357-60.

41. Trapnell C, Williams BA, Pertea G, Mortazavi A, Kwan G, van Baren MJ, Salzberg SL, Wold BJ, Pachter L. Transcript assembly and quantification by RNA-Seq reveals unannotated transcripts and isoform switching during cell differentiation. Nat Biotechnol. 2010;28(5):511-5.

42. Anders $\mathrm{S}$, Huber W. Differential expression analysis for sequence count data. Genome Biol. 2010;11:R16.

43. Young MD, Wakefield MJ, Smyth GK, Oshlack A. Gene ontology analysis for RNA-seq: accounting for selection bias. Genome Biol. 2010;11:R14.

44. Ma C, Wang W, Wang Y, Sun Y, Kang L, Zhang Q, Jiang Y. TMT-labeled quantitative proteomic analyses on the longissimus dorsi to identify the proteins underlying intramuscular fat content in pigs. J Proteome. 2020;213: 103630.

45. Zhu G, Kang L, Wei Q, Cui X, Yang C, Jiang Y. Expression and regulation of MMP-1, MMP-3 and MMP-9 in ovary, follicle and granulosa cells in response to gonadotropins, sex hormones and TGF-b1 in chickens. Biol Reprod. 2014; 90(3):57.

46. Livak KJ, Schmittgen TD. Analysis of relative gene expression data using real-time quantitative $P C R$ and the $2^{-\Delta \Delta C T}$ method. Methods. 2001;25:402-8.

\section{Publisher's Note}

Springer Nature remains neutral with regard to jurisdictional claims in published maps and institutional affiliations.

Ready to submit your research? Choose BMC and benefit from:

- fast, convenient online submission

- thorough peer review by experienced researchers in your field

- rapid publication on acceptance

- support for research data, including large and complex data types

- gold Open Access which fosters wider collaboration and increased citations

- maximum visibility for your research: over $100 \mathrm{M}$ website views per year

At BMC, research is always in progress.

Learn more biomedcentral.com/submissions 